\title{
Identification of genes with altered expression in medullary breast cancer vs. ductal breast cancer and normal breast epithelia
}

\author{
MORTEN F. GJERSTORFF ${ }^{1,4 *}$, VIVIAN M. BENOIT ${ }^{4 *}$, ANNE-VIBEKE LAENKHOLM ${ }^{2}$, \\ OLE NIELSEN $^{2}$, LENE E. JOHANSEN ${ }^{1}$ and HENRIK J. DITZEL ${ }^{1,3,4}$ \\ ${ }^{1}$ Medical Biotechnology Center, Institute of Medical Biology, University of Southern Denmark, Winsloewparken 25; \\ ${ }^{2}$ Departments of Clinical Pathology and ${ }^{3}$ Internal Medicine, Odense University Hospital, Sdr. Boulevard 29, \\ DK-5000 Odense C, Denmark; ${ }^{4}$ Departments of Immunology, The Scripps Research Institute, \\ 10550 North Torrey Pines Road, La Jolla, CA 92037, USA
}

Received December 20, 2005; Accepted February 13, 2006

\begin{abstract}
Medullary breast cancer (MCB) is a morphologically and biologically distinct subtype that, despite cytologically highly malignant characteristics, has a favorable prognosis compared to the more common infiltrating ductal breast carcinoma. MCB metastasizes less frequently, which has been attributed to both immunological and endogenous cellular factors, although little is known about the distinct biology of MCB that may contribute to the improved outcome of MCB patients. To identify candidate genes, we performed gene array expression analysis of cell lines of MCB, ductal breast cancer and normal breast epithelia, and the differential expression of a panel of candidate genes was further validated by quantitative PCR and immunohistochemical analysis of cell lines and tumor biopsies. A limited number of genes, including several members of the GAGE and insulin growth factor binding protein $(I G F B P)$ gene families, Vav1, monoglyceride lipase and NADP(+)dependent malic enzyme, exhibited altered expression in MCB vs. ductal breast cancer, and the differences for some of these genes were confirmed on an extended panel of cell lines by quantitative PCR. Immunohistochemical analysis further established that the expression of monoglyceride lipase was restricted to ductal breast cancer and present in $77 \%$ of these tumors, while Vav1 was restricted to MCB and present in $60 \%$ of tumors. In this study, we have identified genes that are differentially expressed in MCB vs. ductal breast cancer and further analysis of the gene products should illuminate the biological differences between MCB and ductal breast cancer.
\end{abstract}

Correspondence to: Dr Henrik J. Ditzel, Medical Biotechnology Center, Institute of Medical Biology, University of Southern Denmark, Winsloewparken 25, DK-5000 Odense C, Denmark

E-mail: hditzel@health.sdu.dk

${ }^{*}$ Contributed equally

Key words: breast cancer subtypes, DNA microarray, cancer targets

\section{Introduction}

Medullary carcinoma of the breast (MCB) is a morphologically and biologically distinct subtype constituting approximately $3-6 \%$ of all invasive breast cancers. Significant attention has been focused on MCB since this subtype, despite having cytologically highly malignant characteristics, has a favorable prognosis compared to other types of infiltrating breast cancers, including the more common ductal adenocarcinoma $(1,2)$. The 10-year survival for patients with MCB was found to be $84 \%$ compared to $63 \%$ for patients with non-MCB (3-5). Typically, MCB is characterized by wellcircumscribed borders, large and pleomorphic nuclei with prominent nucleoli, numerous mitoses, sparse necrosis, syncytial growth pattern, no tubular component and an intense infiltrate of lymphocytes and plasma cells (1). DNA ploidy studies typically show predominantly aneuploid stem lines, consistent with the high mitotic rates. Risk factors such as lymph node status, tumor size, steroid receptor status, and menopausal status, which are found to be of major prognostic importance in breast cancer in general, have minimal prognostic value in $\mathrm{MCB}$, indicating that MCB is a subtype with unique biological features (6).

Several studies have examined the characteristic lymphoplasmacytic infiltrate in MCB, since it has been hypothesized that these cells react with an antigen directly or indirectly involved in the control of tumor growth. These studies indicate that, while the $\mathrm{T}$ cell immune response may play a role in MCB tumor growth, the specific biological characteristics of MCB cells may be the major factor (7-11). Such biological characteristics include altered expression of genes and signaling pathways, and previous studies have shown that nearly $100 \%$ of MCBs contain alterations in the p53 gene and immunohistochemically verified accumulation of p53 in the nuclei of tumor cells (12-14). In comparison, p53 alterations are found in only 20-40\% of invasive ductal breast cancers. Another study found that MCB was diagnosed much more frequently than expected among biopsy specimens from patients with BRCA1-associated breast cancer (15), a marker otherwise only found in hereditary breast cancer.

DNA microarray has proven to be a useful technique for large-scale gene expression surveys in cancer and other 
Table I. Analysis of members of the IGF system for altered gene expression between MCB and ductal breast cancer using normal breast epithelia cells as a baseline.

\begin{tabular}{|c|c|c|c|c|c|c|}
\hline \multirow{2}{*}{$\begin{array}{l}\text { GenBank } \\
\text { accession number }\end{array}$} & \multirow[b]{2}{*}{ Gene product } & \multicolumn{4}{|c|}{ Sort score } & \multirow{2}{*}{$\begin{array}{l}\text { Affymetrix } \\
\text { probe name }\end{array}$} \\
\hline & & HS578T & MCF7 & BrCa-MZ-01 & MB157 & \\
\hline M35878 & IGFBP-3 & 91.48 & 6.18 & 0.20 & 0.31 & 37319_at \\
\hline U20982 & IGFBP-4 & 13.08 & 30.15 & -5.36 & 0.02 & 39781_at \\
\hline L27560 & IGFBP-5 & 47.30 & 12.47 & 0.06 & 1.36 & 38650_at \\
\hline M62402 & IGFBP-6 & 0 & -10.35 & -11.59 & -8.21 & 1736_at \\
\hline X04434 & IGF receptor I & 0.15 & -5.88 & -0.19 & 0 & 1335_at \\
\hline Y00285 & IGF receptor II & -1.06 & -2.39 & -3.31 & -1.68 & 160027_s_at \\
\hline X57025 & IGF I & -0.01 & 0.03 & 0.09 & 0.01 & 1501_at \\
\hline S73149 & IGF II & -0.22 & -0.25 & -0.14 & -0.11 & 1464_at \\
\hline S62539 & Insulin receptor subtrate-1 & -0.81 & 0.60 & -3.81 & -2.79 & 872_i_at \\
\hline M6439 & Human cyclin D1 & -0.02 & -0.23 & -0.05 & -1.61 & 2017_s_at \\
\hline D31661 & Tyrosine kinase & -0.05 & -0.16 & 3.84 & -0.23 & 2088_s_at \\
\hline
\end{tabular}

Bold text: genes that are up- or down regulated in breast cancer cells of one subtype compared to cells of the other subtype and normal breast epithelial cells.

diseases. Several such microarray studies have analyzed the gene expression profile in breast cancer (16-23), and a gene expression signature consisting of 70 genes has been identified that strongly predicts good or poor prognosis in younger stage I and II breast cancer patients $(21,23)$. The overall 10 -year survival rate was $94.5 \%$ in the good prognosis group, and $54.6 \%$ in the poor prognosis group. These gene expression profiles were better predictors of clinical outcome than current clinical and histological criteria. A second set of reports analyzed expression levels in a larger panel of infiltrating ductal breast cancer and identified a set of genes that could distinguish 5 breast cancer subgroups (luminal A, luminal B, normal breast-like, HER2-overexpressing, and basal-like types); the luminal A subgroup had a favorable prognosis, while the latter two were associated with poor outcomes $(18,24,25)$. Overall, the results from these studies demonstrate the feasibility of cancer classification based solely on gene expression monitoring. Likewise, novel breast cancer-related genes that may play a key role in cancer development and thus serve as therapeutic targets may be identified using this approach. However, none of the studies to date have examined MCB or compared MCB to ductal breast cancer and normal breast epithelia to elucidate differentially regulated pathways between the two breast cancer subtypes.

In this study, we examined the expression levels of more than 7,000 genes in MCB and compared them to the expression levels in infiltrating ductal breast cancer and normal breast epithelia cells to identify genes and pathways involved in the distinct biology of MCB. A panel of candidate genes were identified and further examined by quantitative PCR and immunohistochemistry.

\section{Materials and methods}

Cell lines. The MCB cell line MB-157 (CRL-7721) and the MCF7 (HTB-22), Hs578T (HTB-126), MB231, MB435, MB436 and ZR75.1 ductal breast carcinoma cell lines were purchased from ATCC (Manassas, VA, USA) and cultured under recommended conditions. The MCB cell line BrCaMZ-01 was a gift from Dr V.J. Möbus (University of Ulm, Ulm, Germany) and cultured in Dulbecco's modified Eagle's medium (Invitrogen, Carlsbad, CA, USA) supplemented with $10 \%$ fetal bovine serum, sodium pyruvate, L-glutamine, nonessential amino acids, penicillin $\mathrm{G}$, and streptomycin (26). The human mammary epithelial cells, 184 Birdie (184B; referred to elsewhere in this paper as normal breast epithelial cells), were a gift from Drs Jim Garbe and Martha Stampfer (Lawrence Berkeley National Lab, Berkeley, CA, USA). The 184B cells are derived from organoids isolated from reduction mammoplasty tissues and are finite lifespan, non-tumorigenic cells. The 184B cells were cultured in mammary epithelial growth medium (BioWhittaker, Walkersville, MD, USA) supplemented with $70 \mu \mathrm{g} / \mathrm{ml}$ bovine pituitary extract, $5 \mu \mathrm{g} / \mathrm{ml}$ human transferrin, and $5 \mathrm{mM}$ isoproterenol. The MCF7 line, derived from metastatic ductal adenocarcinoma of a 69year-old Caucasian female, retains several characteristics of differentiated mammary epithelium and is estrogen receptor (ER)-positive, while the Hs578T cell line (HTB-126), derived from a 74-year-old Caucasian female with ductal breast carcinoma, exhibits a stellate cell type and is ER-negative. The MDA-MB-157 ER-negative cell line was derived from an MCB of a 44-year-old black female, and the BrCa-MZ-01 cell line was derived from a primary $\mathrm{MCB}$ of a 61-year-old female and expresses both the progesterone receptor and ER (26). Fresh stocks obtained directly from ATCC or our collaborators were grown under controlled conditions to minimize the passage cycles by the cells, ensuring that they represented the expression profiles of the parental tumor type as much as possible.

Microarray analysis. Total RNA was extracted from two separate preparations of each cell line using the RNeasy Miniprep kit (Qiagen, Valencia, CA, USA) according to the manufacturer's protocol, and the quality of the samples was examined with an Agilent Bioanalyzer (Agilent Technologies, Palo Alto, CA, USA). RNA from each preparation was 
Table II. Analysis of cancer testis antigens for altered gene expression between MCB, ductal breast cancer using normal breast epithelia cells as baseline.

\begin{tabular}{|c|c|c|c|c|c|c|}
\hline \multirow{2}{*}{$\begin{array}{l}\text { GenBank } \\
\text { accession number }\end{array}$} & \multirow[b]{2}{*}{ Gene product } & \multicolumn{4}{|c|}{ Sort score } & \multirow{2}{*}{$\begin{array}{l}\text { Affymetrix } \\
\text { probe name }\end{array}$} \\
\hline & & HS578T & MCF7 & BrCa-MZ-01 & MB157 & \\
\hline L18920 & MAGEA2 & 2.69 & 0.10 & 0.06 & 5.25 & 33518_f_at \\
\hline U03735 & MAGEA3 & 6.81 & -0.14 & -0.15 & 14.67 & 33517_f_at \\
\hline U10688 & MAGEA4 & 0.40 & 0 & 0 & 0.04 & 36302_f_at \\
\hline U10689 & MAGEA5 & 4.65 & 0.02 & 0.04 & 6.42 & 34575_f_at \\
\hline U10693 & MAGEA8 & -0.06 & -0.15 & 0.01 & -0.04 & 34576_at \\
\hline U10694 & MAGEA9 & 0.01 & 0.03 & 0 & 0.01 & 34577_at \\
\hline U10686 & MAGE 11 & 0.60 & 0.27 & 0.10 & -0.11 & 34574_at \\
\hline U93163 & MAGEB2 & -0.03 & -0.08 & 0 & 0 & 35097_at \\
\hline \multicolumn{7}{|l|}{ U19142 } \\
\hline $\begin{array}{l}\text { U1914 } \\
\text { AF055473 }\end{array}$ & GAGE- $1,-2,-8$ & -0.04 & 0.05 & 0 & 0.03 & 31497_at \\
\hline U19144 & GAGE-3 & 0.07 & 0.14 & 20.66 & 0.26 & 31953_f_at \\
\hline \multicolumn{7}{|l|}{ U19142 } \\
\hline U19143 & & 1.35 & 0.11 & 49.52 & 4.41 & 31960_f_at \\
\hline U19145 & GAGE-1, -2, -4, -5, -6, & 0.35 & -0.21 & 47.41 & 2.16 & 33671_f_at \\
\hline U19146 & $-7,-7 \mathrm{~B},-8$ & 0.04 & -0.04 & 42.25 & 2.13 & 37065_f_at \\
\hline U19147 & & -0.16 & 0.14 & 41.62 & 2.38 & 31498_f_at \\
\hline \multicolumn{7}{|l|}{ AF058988 } \\
\hline \multicolumn{7}{|l|}{ AF055473 } \\
\hline \multicolumn{7}{|l|}{ AF055474 } \\
\hline AF058989 & PAGE-1 & 0 & -0.09 & 0 & -0.06 & 32997_at \\
\hline U46193 & RAGE-3 & 16.86 & -2.75 & -1.82 & -3.45 & 41389_s_at \\
\hline U46194 & RAGE-4 & 1.55 & 0 & -0.04 & -0.04 & 1524_at \\
\hline U19180 & BAGE & 0.01 & 0.14 & -0.08 & 0.08 & 1037_at \\
\hline U87459 & NY-ESO-1 & 10.48 & 0.27 & 0.09 & 4.69 & 33637_g_at \\
\hline
\end{tabular}

labeled, hybridized and scanned using standard Affymetrix protocol (27) (protocol available at http://affymetrix.com). Chips were scanned using the Affymetrix ScanArray 3000 using default settings and a target intensity of 250 for scaling. Each RNA preparation was analyzed on HG-U95A gene chips (Affymetrix, Santa Clara, CA, USA), and data was globally normalized by adjusting the mean hybridization intensity for each array to the target intensity of 250 . The mean of the two normalized values for each cell line was used in the subsequent analysis performed with Microsoft Excel. For genes to be selected for further analysis, they should be transcribed in both cell lines of one breast cancer subtype, exhibit a sort score change $>2$ [sort score is a ranking of the fold change and the average difference change (http://www.affymetrix.com)], and not be transcribed or up/down-regulated in the opposite direction in both cell lines of the other subtype and in the normal breast epithelia cell line.

Quantitative RT-PCR. cDNA was generated using Taqman reverse transcription reagents (Applied Biosystems, Foster City, CA, USA) and analyzed by quantitative real-time PCR using SYBR-Green PCR Master Mix (Applied Biosystems) according to the recommendations of the manufacturer. The PCR run consisted of initial denaturation at $95^{\circ} \mathrm{C}$ for $10 \mathrm{~min}$, followed by 45 cycles of annealing at $95^{\circ} \mathrm{C}$ for $15 \mathrm{sec}$ and extension at $60^{\circ} \mathrm{C}$ for $1 \mathrm{~min}$. Reactions were monitored during the extension phase using Sequence Detection System software (Applied Biosystems) and an ABI PRISM ${ }^{\circledR} 7700$ Sequence Detection System (Applied Biosystems).

Relative quantification was performed in triplicate using the standard curve method for relative quantification. The median relative expression levels were normalized with endogenous $\beta$-actin or glyceraldehyde-3-phosphate dehydrogenase (GAPDH) levels, and the normalized relative quantity for each cell line was expressed as the relative fold change compared to the normalized relative quantity of normal breast epithelial cells. The primers for specific amplification of GAGE-4-7B, Vav1, insulin growth factor binding protein 5 (IGFBP-5), GAPDH and ß-actin were: 5'-GAG GGA GCT GTG AGG CAG T-3' (GAGE-4-7B sense), 5'-CAT TTC AGG AGG CTG TAC AT-3' (GAGE-4-7B antisense), 5'AGC AGT GGG AAG CAC AAA GTA TT-3' (Vav1 sense), 
Table III. Analysis of enzymes involved in lipid synthesis and of a hematopoietic signaling molecule for altered gene expression between MCB, ductal breast cancer using normal breast epithelia cells as baseline.

\begin{tabular}{llcccrr}
\hline \multirow{2}{*}{$\begin{array}{l}\text { GenBank } \\
\text { accession number }\end{array}$} & Gene product & HS578T & MCF7 & BrCa-MZ-01 & MB157 & $\begin{array}{r}\text { Affymetrix } \\
\text { probe name }\end{array}$ \\
\cline { 3 - 6 } X16316 & Vav1 proto-oncogene & 0.09 & 0.50 & $\mathbf{8 . 2 8}$ & $\mathbf{2 . 0 5}$ & 1919_at \\
U43944 & $\begin{array}{l}\text { Breast cancer cytosolic } \\
\text { NADP+-dependent malic enzyme }\end{array}$ & -1.65 & -1.54 & $\mathbf{- 5 1 . 0 1}$ & $\mathbf{- 1 7 . 0 5}$ & 837_s_at \\
U67963 & Monoglyceride lipase & $\mathbf{2 4 . 6 6}$ & $\mathbf{5 . 1 8}$ & -1.08 & -0.88 & 35792_at \\
\hline
\end{tabular}

5'-GTC ACG GGC GCA GAA GTC-3' (Vav1 antisense), 5'TGC TCA ATC TTC ATG AGA CAA AGG-3' (IGFBP-5 sense), 5'-GCT GAC TCG GCA GGT CAA G-3' (IGFBP-5 antisense), 5'-TGC ACC ACC AAC TGC TTA GC-3' (GAPDH sense), 5'-GGC ATG GAC TGT GGT CAT GAG3' (GAPDH antisense), 5'-AGC CTC GCC TTT GCC GA-3' (B-actin sense) and 5'-CTG GTG CCT GGG GCG-3' (ß-actin antisense). For amplication of monoglyceride lipase (MGL) and NADP(+)-dependent malic enzyme, pre-designed RT2 PCR probes (MedProbe, Lund, Sweden) were used. The quantifications were performed twice in their entirety, and the similar relative fold changes confirmed reproducibility of the methods.

Immunohistochemistry and immunocytochemistry. Plasma/ thrombin cell blocks were generated from the cell lines by adding $50 \mu 1$ of plasma to $5 \times 10^{5}$ cells followed by $35 \mu 1$ of bovine thrombin (Biofac A/S, Ejby, Denmark), which leads to the formation of a clot surrounding the cells. The plasma/ thrombin cell clots and patient tissue biopsies were fixed in $4 \%$ formaldehyde, $\mathrm{pH} 7.4$ for $24 \mathrm{~h}$. Sections were cut, deparaffinized, treated with $1.5 \% \mathrm{H}_{2} \mathrm{O}_{2}$ in Tris-buffered saline, $\mathrm{pH} 7.5$ for 10 min to block endogenous peroxidase activity, rinsed in distilled $\mathrm{H}_{2} \mathrm{O}$, demasked and washed in TNT buffer ( $0.1 \mathrm{M}$ Tris, $0.15 \mathrm{M} \mathrm{NaCl}, 0.05 \%$ Tween-20, $\mathrm{pH}$ 7.5). Sections were subsequently incubated with either polyclonal rabbit anti-IGFBP-5 antibody (\#06-110; Upstate, Charlottesville, VA, USA), polyclonal rabbit anti-Vav1 antibody (\#2502; Cell Signaling, Beverly, MA, USA), polyclonal mouse anti-GAGE antibody (produced in-house) or polyclonal rabbit anti-monoglyceride lipase antibodies (\#100035; Cayman Chemical, Ann Arbor, MI, USA) diluted in antibody diluent (S2022; Dako, Glostrup, Denmark) for $1 \mathrm{~h}$ at room temperature. Polyclonal mouse anti-GAGE antibody was produced by the immunization of mice with a full-length recombinant GAGE-7-GST antigen produced in E. coli (unpublished data). Sections were washed with TNT and incubated with horse-radish peroxidase conjugated 'Readyto-Use' EnVision+ ${ }^{\mathrm{TM}}$ polymer (K4003 for rabbit antibodies and K4001 for mouse antibodies; Dako) for $30 \mathrm{~min}$, followed by another wash with TNT. The final reaction product was visualized by incubating with 3,3'-diaminobenzidine $(\mathrm{DAB})^{+}$ substrate-chromogen for $10 \mathrm{~min}$, followed by washing with $\mathrm{H}_{2} \mathrm{O}$ and the counterstaining of sections with Mayer's hematoxylin before mounting in AquaTex (Merck Inc.,
Whitehouse Station, NJ, USA). For each experiment, isotype matched or no primary antibody was included as a control.

\section{Results}

Microarray analysis. To identify genes with altered expression and pathways in MCB that may be involved in the distinct biology of MCB and might be attributed to a more favorable prognosis compared to ductal breast cancer, we analyzed the gene expression profiles of MCB, ductal breast cancer and normal breast epithelia cell lines. RNA purified from the only two MCB cell lines currently available (MB-157 and BrCa-MZ-01), two ductal breast cancer cell lines (MCF7 and HS578T), and the finite lifespan normal human breast epithelial cell line (184B) was used for microarray analysis using the Affymetrix HG-U95A high density GeneChip, which contains 12,599 probe sets for 7,598 human genes (raw data available from ArrayExpress database with accession no. E-MEXP-441). Two RNA preparations from each cell line were analyzed on separate HG-U95A GeneChips to evaluate the reproducibility of results. Analysis of the two sets demonstrated that the data were reproducible, and the mean of the two values was consequently used in subsequent analysis. Using normal breast epithelia cells as a baseline, we identified 399 genes of which the expression levels were altered 2-fold or more in terms of sort score in both MCB cell lines. Similarly, we identified 234 genes of which the expression levels were altered 2-fold or more in both ductal breast cancer cell lines compared to normal breast epithelial cells. Combining the two data sets, we identified 11 genes of which the expression levels were altered in one breast cancer subtype compared to normal breast epithelia and unchanged or altered in the opposite direction in both cell lines of the other subtype (Tables I-III).

Expression of IGFBP-3, -4 and -5 , belonging to the 6 member IGFBP family, was found to be up-regulated in both ductal cancer cell lines, while the expression level in both MCB cell lines was similar to that observed in normal breast epithelia (Table I). Since IGFBPs bind with high affinity to insulin-like growth factors (IGF) and regulate access of the IGF ligands to IGF receptors, other members of the IGF system could be up- or down-regulated, perhaps as a compensatory mechanism (Table I). However, with the exception of insulin receptor substrate 1 (IRS-1), no consistent changes were observed within the breast cancer subtypes. IRS-1 plays an 

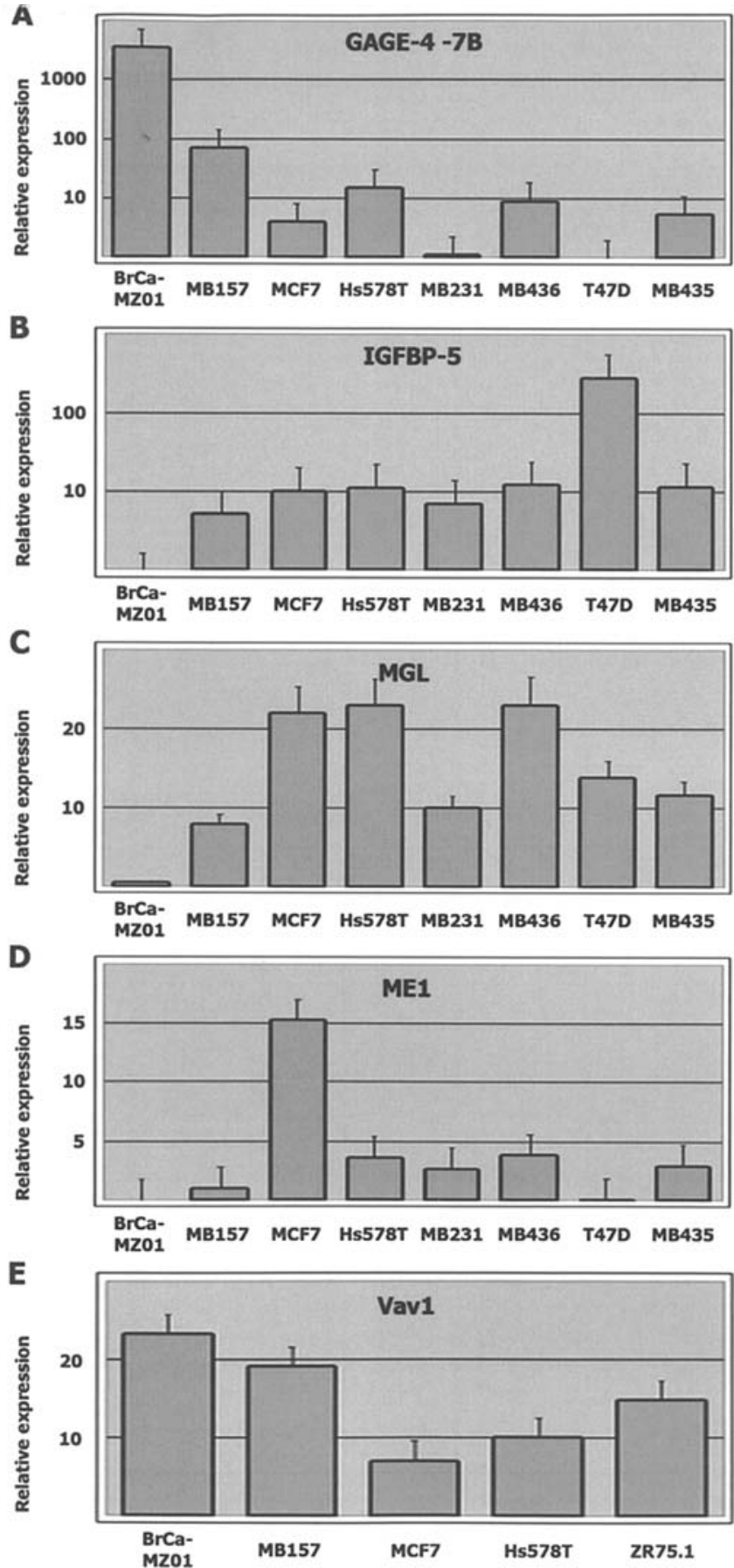

Figure 1. Verification of gene expression differences between MCB, ductal breast cancer and normal breast epithelial cells as determined by quantitative real-time PCR analysis. Histograms are expressed as the fold change compared to normal breast epithelial cells (184B). Error bars represent a $95 \%$ confidence interval for the mean. (A) Increased GAGE-4$7 \mathrm{~B}$ gene expression in MCB versus ductal breast cancer and normal breast epithelial cells. (B) Increased IGFBP-5 gene expression in ductal breast cancer versus MCB and normal breast epithelial cells. (C) Increased MGL gene expression in ductal breast cancer versus MCB and normal breast epithelial cells. (D) Increased cytosolic NADP(+)-dependent malic enzyme (ME1) gene expression in ductal breast cancer versus MCB and normal breast epithelial cells. (E) Increased Vav1 gene expression in MCB versus ductal breast cancer and normal breast epithelial cells.

important role in mediating apoptosis, cell differentiation and cell transformation, and has been shown to be constitutively activated in a variety of solid tumors, including breast cancer. IRS-1 was down-regulated in the MCB cell lines, BrCa-MZ-01
Table IV. Immunohistochemical analysis of candidate protein expression in medullary and ductal breast cancer tumor biopsies.

\begin{tabular}{lcc}
\hline $\begin{array}{l}\text { Candidate } \\
\text { protein }\end{array}$ & $\begin{array}{c}\text { Medullary breast } \\
\text { cancer }(\mathrm{n}=5)\end{array}$ & $\begin{array}{r}\text { Ductal breast } \\
\text { cancer }(\mathrm{n}=22)\end{array}$ \\
\hline GAGE-1-8 & $40 \%(2)$ & $9 \%(2)$ \\
IGFBP-5 & $0 \%(0)$ & $18 \%(4)$ \\
Vav1 & $60 \%(3)$ & $0 \%(0)$ \\
Monoglyceride lipase & $0 \%(0)$ & $77 \%(17)$ \\
\hline
\end{tabular}

and MB157, compared to the ductal cell lines, MCF7 and HTB126, and normal breast epithelia.

The expression of a second group of genes, GAGE-4-7B, members of the GAGE gene family of the group of testis cancer antigens, was strongly up-regulated in both MCB cell lines, but absent in cell lines of ductal cancer or normal epithelia (Table II). GAGE has previously been shown to be present in cancers of different origin, but has not been evaluated in MCB or other breast cancer subtypes. Other cancer testis antigens, including members of the MAGE and RAGE gene families and NY-ESO-1, were expressed in some of the cell lines, but not found to be selectively expressed by any of the breast cancer subtypes (Table II).

Vav1, a multi-pathway signaling molecule normally present only in the hematopoietic compartment (28), was strongly up-regulated in both MCB cell lines, but absent in ductal cancer and normal breast epithelial cells (Table III). Interestingly, a recent report has also found Vav1 expression in approximately half of pancreatic cancers, where it was found to be associated with tumor cell proliferation (29).

Two genes that encode enzymes involved in lipid metabolism, i.e. MGL (30), and cytosolic $\mathrm{NADP}^{+}$-dependent malic enzyme (31), were also shown to have altered expression levels in MCB vs. ductal breast cancer (Table III). MGL, a serine hydrolase shown to increase tumor cell invasion (32) and proliferation (33) by hydrolyzing 2-arachidonoylglycerol, was highly expressed in the MCF7 and HS578T ductal cell lines, but not in the MCB cell lines BrCa-MZ-01 and MB157, or normal breast epithelial cells. NADP+-dependent malic enzyme, which has been shown to catalyze the NADPlinked oxidative decarboxylation of malate to pyruvate and $\mathrm{CO}_{2}$ (34) and has been implicated in providing the reducing power of NADPH to the cytosol for fatty acid synthesis from acetyl CoA $(35,36)$, was down-regulated in the MCB cell lines.

Quantitative PCR analysis. To verify the gene alterations observed in the microarray experiments, we carried out quantitative determination of gene expression changes by real-time PCR. In addition to the MCB and ductal breast cancer cells lines included in the gene array analysis, a panel of other relevant ductal breast cancer cell lines were examined by quantitative PCR, as shown in Fig. 1. Since the two MCB cell lines tested are the only available cell lines of this breast cancer subtype, no additional MCB cell lines could be included in the validation study. The quantitative PCR for GAGE-4-7B confirmed that GAGE-4-7B was highly expressed in MCB 

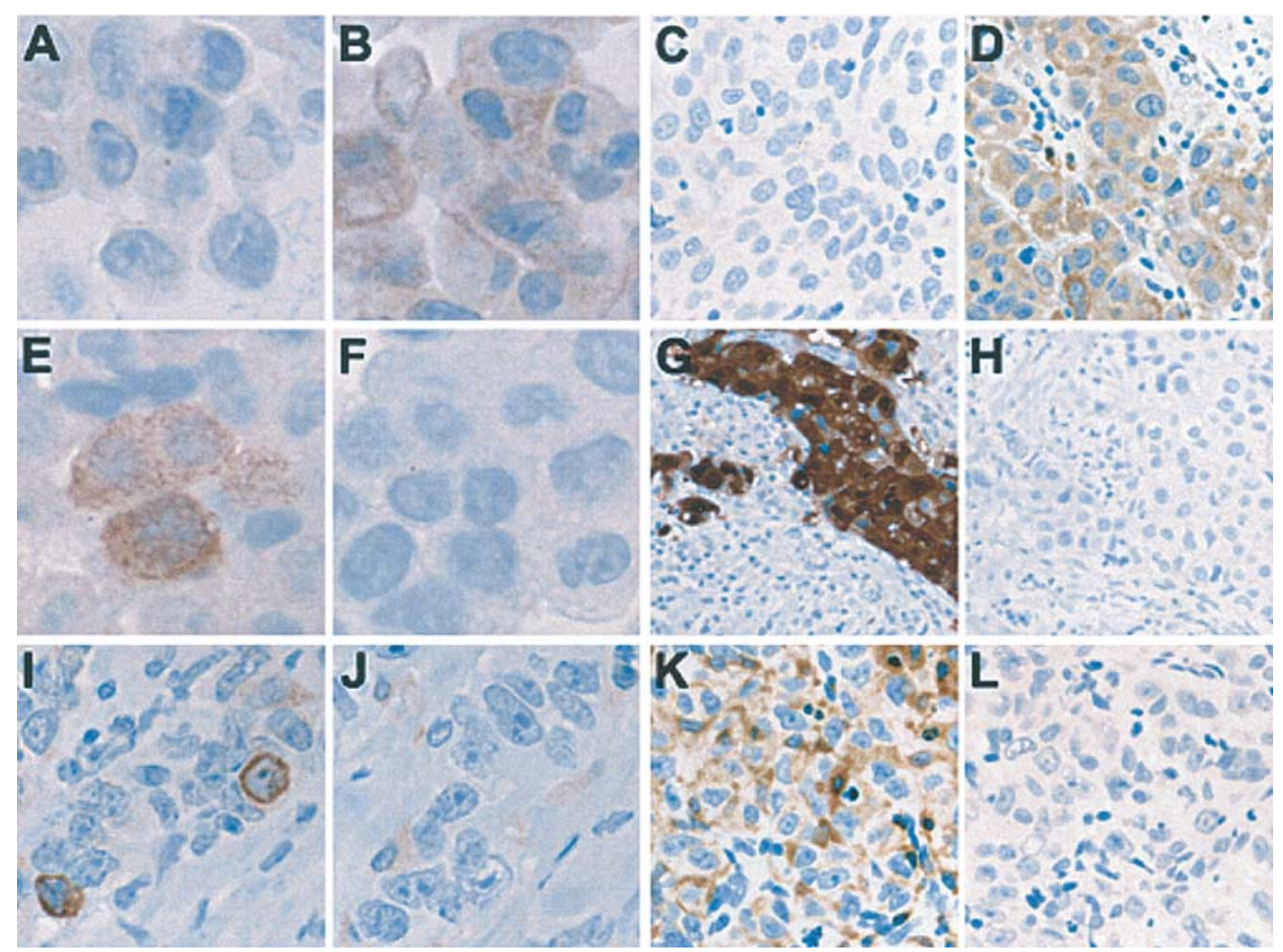

Figure 2. Immunocytochemical and immunohistochemical analysis of the expression of 4 gene products that exhibited altered gene expression in the microarray analysis between ductal breast cancer, MCB and normal breast epithelia. In agreement with the DNA microarray data, MGL was not present in the MCB cell line, MB157 (A), but was present in the ductal breast cancer cell line, Hs578T (B). Further, MGL was not found in any of 5 MCB tumors tested (C) but was found in some ductal breast cancers (D). GAGE, detected with a mouse polyclonal anti-GAGE antibody, was found in the MCB cell line, BrCa-MZ-01 (E), but not in the ductal breast cancer cell line, MCF7 (F). However, GAGE was not restricted to MCB tumors, it was also found in a few ductal breast cancer biopsies $(\mathrm{G})$. Mouse sera obtained prior to GAGE immunization verified that the GAGE staining was specific (H). IGFBP was observed in some ductal breast cancers (I) but not in MCB (L) or normal breast epithelia. In the IGFBP-positive ductal cancers, IGFBP staining was confined to individual cancer cells, while the majority of cancer cells were IGFBP-negative. The adjacent section stained with an anti-Vav1 antibody exhibited no binding, verifying the specificity of the anti-IGFBP staining (J). In contrast, the anti-Vav1 antibody stained MCB biopsies (K), which were not stained with an anti-IGFBP-5 antibody (L). The cell lines and the tissue specimens were formalin-fixed and paraffin-embedded.

compared to ductal breast cancer and normal breast epithelial cells, and showed a 2,200-fold increase in GAGE-4-7B expression in $\mathrm{BrCa}-\mathrm{MZ}-01$ (Fig. 1A). In addition, realtime PCR showed that only very low expression levels of GAGE-4-7B existed in 6 ductal breast cancer patient tissue specimens (data not shown). Quantitative PCR analysis revealed increased IGFBP-5 (Fig. 1B), MGL (Fig. 1C) and cytosolic NADP(+)-dependent malic enzyme (Fig. 1D) expression levels in ductal breast cancer cells compared to MCB or normal breast epithelial cells. Furthermore, the difference in expression levels was even higher with realtime PCR than microarray analysis, probably due to the higher accuracy of real-time quantitative PCR. Finally, as shown in Fig. 1E, high expression of Vav1 was observed in MCB relative to ductal breast cancer or normal breast epithelia.

Protein expression analysis. To verify that the observed gene expression alterations correlated with alterations at the protein level, immunocytochemical analysis of the different cell lines used for the DNA microarray study was performed. Staining with an anti-MGL antibody was observed in both ductal breast cancer cell lines but not in MCB cells (Fig 2A and B). The staining was localized to the cell surface and cytoplasm. On the contrary, the ductal cell lines showed no staining with an anti-IGFBP-5 antibody. However, this is not surprising since IGFBP-5 is a secreted protein. As no antiGAGE antibodies were commercially available, we immunized mice with full-length recombinant GAGE-7. By Western blotting and ELISA, the antibody was shown to specifically recognize conserved areas of GAGE members (unpublished data). Using immunohistochemistry, the polyclonal anti-GAGE antibody was shown to stain testis but not 24 other normal tissues, including those of the spleen, tonsil, skin, thymus, lung, esophagus, parotid gland, rectum, intestine, ventricle, liver, cerebellum, muscle, kidney, bladder and thyroid. The GAGE antibody was found to stain a subpopulation of the MCB BrCa-MZ-01 cells but not the ductal cell lines (Fig. 2E and F). Although the MCB cell line, MB157, was shown by microarray and real-time PCR to express GAGE, the cell line was not stained by the GAGE antibody, presumably due to a low expression level. The cell lines were also examined for staining with a polyclonal anti-Vav1 antibody but, surprisingly, no staining was observed. This may also be explained by low expression levels.

Expression of the candidate genes at the protein level was also evaluated in a small number of breast tumors surgically removed from patients (Table IV). A thorough examination of the expression specificity of candidate genes for a given 
breast cancer subtype by examining a large number of breast cancer biopsies was beyond the scope of this study. The staining of breast cancer biopsies revealed MGL staining in 17 of 22 ductal breast cancers (Fig. 2D), but not in 5 MCBs (Fig. 2C) or normal breast epithelia. In some ductal breast tumors, all cancer cells exhibited cytoplasmic staining, as depicted in Fig. 2D, while less than $40 \%$ were stained by the antibody in others. The 27 breast cancers were also tested for IGFBP-5 staining. As shown in Fig. 2I, IGFBP-5 staining was observed in 4 of the ductal breast cancers, but not in MCB or normal breast epithelia (Fig. 2L). In the IGFBP-5positive ductal cancers, staining was confined to individual cancer cells, while the majority of cancer cells were IGFBP-5negative. However, immunohistochemistry is not an optimal technique for detecting IGFBP-5 expression since IGFBP-5 is a secreted protein. Examination of GAGE expression in the 27 breast cancer biopsies showed staining in 2 of 5 MCB cancers and 2 of 22 ductal breast cancers (Fig. 2G), demonstrating that GAGE expression was not limited to the MCB subtype. Mouse sera obtained prior to immunization with GAGE were used as controls in all experiments and verified the specificity of GAGE staining (Fig. 2H). Analysis of breast cancers with the polyclonal anti-Vav1 antibody revealed staining in 3 of $5 \mathrm{MCB}$ tumor biopsies (Fig. 2K), while no staining was observed in the 22 ductal breast cancers. In all tissue sections, intense staining of cells of hematopoietic origin was observed with the anti-Vav1 antibody.

\section{Discussion}

Several morphological studies of MCB have been undertaken to illuminate its distinct biology and define the mechanisms that may contribute to the favorable prognosis of this breast cancer subtype compared to the more common infiltrating ductal breast cancer. In this study, we used gene array combined with quantitative PCR to examine the gene expression profile of MCB cell lines and compare it to those of ductal breast cancer and normal breast epithelial cells, thereby identifying genes with altered expression. Such candidate genes may yield clues about disease pathogenesis, be used for genetic classification of cancer subtypes, and ultimately identify new targets for breast cancer therapy. Of more than 7,000 genes surveyed, only a limited number of genes exhibited altered expression profiles in the gene chip analysis and met the relatively strict inclusion criteria. These were members of the IGF family, cancer testis antigens, enzymes involved in lipid synthesis and a hematopoietic signaling molecule.

Three IGFBPs, IGFBP-3, -4 and -5, which have been shown to play important roles as regulatory proteins in ductal breast cancer (37), were shown to be up-regulated in ductal breast cancer compared to MCB or normal breast epithelia. In agreement with this, others have found that IGFBP-5 is not expressed in normal breast epithelial cells $(38,39)$. However, IGFBP-5 is expressed in ductal breast cancer and normal early development of the breast, where it is important in mammary gland remodeling, which shares features with cell invasion $(38,40)$. Since IGFBPs have been shown to be under transcriptional control of the ER, it could be speculated that the differences were a result of ER status, but because both the MCB and ductal breast cancer groups contained one $\mathrm{ER}^{+}$and one $\mathrm{ER}^{-}$cell line, this explanation is unlikely. IGFBP-5 was found to be overexpressed in metastases when paired samples of primary tumors and corresponding metastases were examined by microarray (39). IGFBP-5 was also identified as one of the 70 signature genes correlating with poor survival in patients with breast carcinoma (21). IGFBPs can affect cell function in an IGF-dependent or -independent manner, and IGFBP-5 expression has also been shown to activate PI-3 kinase (41) and enhance the survival of breast carcinoma cells (42). Thus, the normal level of IGFBP-5 in MCB may be partially responsible for the low level of metastasis observed in patients with this breast cancer subtype. Further studies will determine whether IGFBP-5 is a potential target for the prevention of breast carcinoma metastases.

MGL was also shown to be highly expressed in ductal breast cancer compared to MCB or normal breast epithelia by microarray analysis. This was confirmed by IHC, where 17 of 20 ductal breast cancers were MGL-positive. MGL inactivates 2-arrachidoylglycerol (37), which has been shown to inhibit invasion (32) and exert anti-proliferative activity on several cancer cell lines, including prostate, breast, and colon cancers as well as gliomas $(33,43)$. Further, a comparative microarray analysis of metastatic and non-metastatic variants (nude mouse system) derived from the MB-435 human breast carcinoma cell line showed that high MGL gene expression was associated with the metastatic phenotype (44). The combined data suggest that MGL may be an important enzyme in the metastatic process through its effect on invasion and proliferation, and might be partially responsible for the lower frequency of metastasis in patients with MCB vs. ductal breast cancers.

Our microarray analysis and quantitative PCR also indicated that the cytosolic NADP(+)-dependent malic enzyme was down-regulated in MCB compared to ductal breast cancer and normal breast epithelia. However, the lack of an antibody to cytosolic NADP(+)-dependent malic enzyme negated further examination of expression at the protein level by immunohistochemisty. A study comparing gene expression in breast cancer cells in quiescent and late G1 phase of the cell cycle showed that mRNA of NADP(+)-dependent malic enzyme were specifically expressed in quiescence (45). Interestingly, the mitochondrial isoform of $\mathrm{NADP}(+)$-dependent malic enzyme has been found to play a crucial role in the glutamine metabolism for energy production in rapidly dividing cells and tumors, and to be up-regulated in these cells (31). Similar analysis of the cytosolic isoform of NADP(+)-dependent malic enzyme in different cancer cells has not been undertaken.

GAGE-4-7B genes were found to be significantly upregulated in both MCB cell lines compared to ductal breast cancer and normal breast epithelia cells by both microarray analysis and real-time PCR. With the exception of testis, GAGE genes that are not expressed in normal tissues have been found in a variety of cancers, including melanoma, lung and esophageal carcinomas, but not in colorectal or renal carcinomas (46-49). To our knowledge, GAGE has never been examined systematically in breast cancer. GAGE has previously been shown to elicit strong CTL responses in melanoma, suggesting that GAGE in MCB and other GAGEexpressing breast tumors may serve as a target of the tumorinfiltrating CTL response (49). The examination of GAGE 
expression at the protein level using an anti-GAGE antibody demonstrated GAGE in the BrCa-MZ-01 MCB cell line but not in the two ductal breast cancer cell lines, MCF7 and HS578T. Only approximately 5\% of BrCa-MZ-01 cells were stained by the GAGE antibody, indicating that GAGE expression is related to a distinct population that exists within the cell line. The nature of the heterogeneous expression of GAGE is currently being investigated. Surprisingly, the MB157 MCB cell line was not stained by the polyclonal GAGE antibody, perhaps due to expression levels lower than the detection limit of the antibody. The examination of an extended panel of ductal breast cancer biopsies demonstrated that GAGE is also expressed in specimens of this subtype, indicating that GAGE is not a selective marker of MCB.

Microarray analysis also revealed Vav1 to be selectively expressed in MCB cell lines, and these results were confirmed by quantitative PCR. Vav1, originally identified on the basis of its transforming potential, is normally only expressed in the hematopoietic compartment, but reports have identified Vav1 in pancreatic cancer associated with tumor cell proliferation $(28,29)$. Surprisingly, Vav1 protein was not detected in any of the cell lines, though it was detected in 3 of 5 MCB biopsies.

During the preparation of this study, a report showed that overexpression of cyclin $\mathrm{E}$ in a breast cancer cell line promotes increased adhesive properties, decreased motility and invasive potential (50). Furthermore, it was found that upregulation of cyclin $\mathrm{E}$ was significantly associated with $\mathrm{MCB}$ compared to other breast cancer subtypes. Collectively, these results indicate that cyclin $\mathrm{E}$ may contribute to the distinct biology of MCB. In our microarray study, cyclin E was found to be up-regulated only in the MB157 MCB cell line, while the level in the BrCa-MZ01 MCB cell line and ductal breast cancer cell lines was similar to that of normal breast epithelial cells.

The use of cell lines, as examined in this study, ensures the consistency of the RNA and confirms that the obtained gene expression profiles reflect only cancer cells and not contaminating connective tissue, infiltrating lymphocytes or vascular cells, a common interpretation problem when whole cancer tissue samples from patients are used as an RNA source. Thus, these cell lines may provide a good baseline. However, since a network of physical and biochemical signals, including the adhesive, growth factor, and hormonal milieu, influence gene expression in diverse tissues, differences between the gene expression profile of cells in cell cultures and those found 'in context,' i.e. within a proper tissue structure, are likely to be observed. It is therefore important to extensively validate the observed differences using tumor biopsies at both the genomic and proteomic level. The examination of a larger panel of MCB cell lines would have been preferable, but the two MCB cells lines in this study are, to our knowledge, the only ones available.

Our microarray analysis of a panel of breast cell lines and a normal breast epithelial cell line identified candidate genes that exhibited altered expression in MCB compared to ductal breast cancer and normal epithelia. Analysis at the protein level on a panel of breast tumors demonstrated that only some of these candidate genes were consistently and differentially expressed in MCB versus ductal breast cancer. Further analysis of these candidate genes may reveal characteristics that shape the distinct biology of these two breast cancer subtypes. In order to validate the identified genes and possibly identify other genes differentiating MCB from ductal breast cancer, we have initiated a gene microarray analysis of a large panel of MCB and ductal breast cancer biopsies.

\section{Acknowledgements}

We would like to thank Steve Head and Sabine Kurz-Camacho for advice and assistance with analysis of the microarray data and the real-time RT-PCR, and M.K. Occhipinti-Bender for editorial assistance. This study was supported by the Danish Cancer Society, Danish Cancer Research Foundation, California Breast Cancer Foundation, LeoPharma Research Foundation, and Dagmar Marshall Foundation.

\section{References}

1. Ridolfi RL, Rosen PP, Port A, Kinne D and Mike V: Medullary carcinoma of the breast: a clinicopathologic study with 10-year follow-up. Cancer 40: 1365-1385, 1977.

2. Rapin V, Contesso G, Mouriesse H, et al: Medullary breast carcinoma. A re-evaluation of 95 cases of breast cancer with inflammatory stroma. Cancer 61: 2503-2510, 1988.

3. Wargotz ES and Silverberg SG: Medullary carcinoma of the breast: a clinicopathologic study with appraisal of current diagnostic criteria. Hum Pathol 19: 1340-1346, 1988.

4. Pedersen L, Zedeler K, Holck S, Schiodt T and Mouridsen HT: Medullary carcinoma of the breast, proposal for a new simplified histopathological definition. Based on prognostic observations and observations on inter- and intraobserver variability of 11 histopathological characteristics in 131 breast carcinomas with medullary features. Br J Cancer 63: 591-595, 1991.

5. Ellis IO, Galea M, Broughton N, Locker A, Blamey RW and Elston CW: Pathological prognostic factors in breast cancer. II. Histological type. Relationship with survival in a large study with long-term follow-up. Histopathology 20: 479-489, 1992 .

6. Pedersen L, Zedeler K, Holck S, Schiodt T and Mouridsen HT: Medullary carcinoma of the breast. Prevalence and prognostic importance of classical risk factors in breast cancer. Eur J Cancer 31A: 2289-2295, 1995.

7. Gottlinger HG, Rieber P, Gokel JM, Lohe KJ and Riethmuller G: Infiltrating mononuclear cells in human breast carcinoma: predominance of $\mathrm{T}^{+}$monocytic cells in the tumor stroma. Int $\mathrm{J}$ Cancer 35: 199-205, 1985.

8. Mathoulin MP, Xerri L, Jacquemier J, Adelaide J, Parc P and Hassoun J: Unrestricted T-cell receptor V-region gene repertoire in tumor-infiltrating lymphocytes from human breast carcinomas. Cancer 72: 506-510, 1993.

9. Yakirevich E, Izhak OB, Rennert G, Kovacs ZG and Resnick MB: Cytotoxic phenotype of tumor infiltrating lymphocytes in medullary carcinoma of the breast. Mod Pathol 12: 1050-1056, 1999.

10. Hansen MH, Nielsen HV and Ditzel HJ: Translocation of an intracellular antigen to the surface of medullary breast cancer cells early in apoptosis allows for an antigen-driven antibody response elicited by tumor-infiltrating B cells. J Immunol 169: 2701-2711, 2002

11. Hansen MH, Nielsen $\mathrm{H}$ and Ditzel HJ: The tumor-infiltrating B cell response in medullary breast cancer is oligoclonal and directed against the autoantigen actin exposed on the surface of apoptotic cancer cells. Proc Natl Acad Sci USA 98: 12659-12664, 2001.

12. Jensen V, Jensen ML, Kiaer H, Andersen J and Melsen F: MIB-1 expression in breast carcinomas with medullary features. An immunohistological study including correlations with p53 and bcl-2. Virchows Arch 431: 125-130, 1997.

13. Domagala W, Harezga B, Szadowska A, Markiewski M, Weber K and Osborn M: Nuclear p53 protein accumulates preferentially in medullary and high-grade ductal but rarely in lobular breast carcinomas. Am J Pathol 142: 669-674, 1993. 
14. Marchetti A, Buttitta F, Pellegrini S, et al: p53 mutations and histological type of invasive breast carcinoma. Cancer Res 53: 4665-4669, 1993.

15. Eisinger F, Jacquemier J, Charpin C, et al: Mutations at BRCA1: the medullary breast carcinoma revisited. Cancer Res 58: 1588-1592, 1998 .

16. Sgroi DC, Teng S, Robinson G, LeVangie R, Hudson JR Jr and Elkahloun AG: In vivo gene expression profile analysis of human breast cancer progression. Cancer Res 59: 5656-5661, 1999.

17. Perou CM, Jeffrey SS, van de Rijn M, et al: Distinctive gene expression patterns in human mammary epithelial cells and breast cancers. Proc Natl Acad Sci USA 96: 9212-9217, 1999.

18. Perou CM, Sorlie T, Eisen MB, et al: Molecular portraits of human breast tumours. Nature 406: 747-752, 2000.

19. Hedenfalk I, Duggan D, Chen Y, et al: Gene-expression profiles in hereditary breast cancer. N Engl J Med 344: 539-548, 2001

20. West M, Blanchette C, Dressman H, et al: Predicting the clinical status of human breast cancer by using gene expression profiles. Proc Natl Acad Sci USA 98: 11462-11467, 2001.

21. van't Veer LJ, Dai H, van de Vijver MJ, et al: Gene expression profiling predicts clinical outcome of breast cancer. Nature 415 : 530-536, 2002.

22. Dressman MA, Baras A, Malinowski R, et al: Gene expression profiling detects gene amplification and differentiates tumor types in breast cancer. Cancer Res 63: 2194-2199, 2003.

23. van de Vijver MJ, He YD, van't Veer LJ, et al: A geneexpression signature as a predictor of survival in breast cancer. N Engl J Med 347: 1999-2009, 2002.

24. Sorlie T, Perou CM, Tibshirani R, et al: Gene expression patterns of breast carcinomas distinguish tumor subclasses with clinical implications. Proc Natl Acad Sci USA 98: 10869-10874, 2001.

25. Sorlie T, Tibshirani R, Parker J, et al: Repeated observation of breast tumor subtypes in independent gene expression data sets. Proc Natl Acad Sci USA 100: 8418-8423, 2003.

26. Mobus VJ, Moll R, Gerharz CD, et al: Differential characteristics of two new tumorigenic cell lines of human breast carcinoma origin. Int J Cancer 77: 415-423, 1998.

27. Lockhart DJ, Dong H, Byrne MC, et al: Expression monitoring by hybridization to high-density oligonucleotide arrays. Nat Biotechnol 14: 1675-1680, 1996.

28. Katzav S, Martin-Zanca D and Barbacid M: vav, a novel human oncogene derived from a locus ubiquitously expressed in hematopoietic cells. EMBO J 8: 2283-2290, 1989.

29. Fernandez-Zapico ME, Gonzalez-Paz NC, Weiss E, et al: Ectopic expression of VAV1 reveals an unexpected role in pancreatic cancer tumorigenesis. Cancer Cell 7: 39-49, 2005.

30. Dinh TP, Freund TF and Piomelli D: A role for monoglyceride lipase in 2-arachidonoylglycerol inactivation. Chem Phys Lipids 121: 149-158, 2002.

31. Moreadith RW and Lehninger AL: The pathways of glutamate and glutamine oxidation by tumor cell mitochondria. Role of mitochondrial NAD(P)+-dependent malic enzyme. J Biol Chem 259: 6215-6221, 1984

32. Nithipatikom K, Endsley MP, Isbell MA, et al: 2-arachidonoylglycerol: a novel inhibitor of androgen-independent prostate cancer cell invasion. Cancer Res 64: 8826-8830, 2004.

33. Melck D, De Petrocellis L, Orlando P, et al: Suppression of nerve growth factor Trk receptors and prolactin receptors by endocannabinoids leads to inhibition of human breast and prostate cancer cell proliferation. Endocrinology 141: 118-126, 2000 .
34. Ochoa S, Veiga Salles JB and Ortiz PJ: Biosynthesis of dicarboxylic acids by carbon dioxide fixation. III. Enzymatic synthesis of iota-malic acid by reductive carboxylation of pyruvic acid. J Biol Chem 187: 863-874, 1950.

35. Tepperman HM and Tepperman J: Patterns of dietary and hormonal induction of certain Nadp-linked liver enzymes. Am J Physiol 206: 357-361, 1964.

36. Wise EM and Ball EG Jr: Malic enzyme and lipogenesis. Proc Natl Acad Sci USA 52: 1255-1263, 1964.

37. Sachdev D and Yee D: The IGF system and breast cancer. Endocr Relat Cancer 8: 197-209, 2001.

38. Wood TL and Yee D: Introduction: IGFs and IGFBPs in the normal mammary gland and in breast cancer. J Mammary Gland Biol Neoplasia 5: 1-5, 2000.

39. Hao X, Sun B, Hu L, et al: Differential gene and protein expression in primary breast malignancies and their lymph node metastases as revealed by combined cDNA microarray and tissue microarray analysis. Cancer 100: 1110-1122, 2004.

40. Tonner E, Barber MC, Travers MT, Logan A and Flint DJ: Hormonal control of insulin-like growth factor-binding protein-5 production in the involuting mammary gland of the rat. Endocrinology 138: 5101-5107, 1997.

41. Miyake H, Nelson C, Rennie PS and Gleave ME: Overexpression of insulin-like growth factor binding protein-5 helps accelerate progression to androgen-independence in the human prostate LNCaP tumor model through activation of phosphatidylinositol 3'-kinase pathway. Endocrinology 141: $2257-2265,2000$

42. McCaig C, Perks CM and Holly JM: Intrinsic actions of IGFBP-3 and IGFBP-5 on Hs578T breast cancer epithelial cells: inhibition or accentuation of attachment and survival is dependent upon the presence of fibronectin. J Cell Sci 115: 4293-4303, 2002.

43. Ligresti A, Bisogno T, Matias I, et al: Possible endocannabinoid control of colorectal cancer growth. Gastroenterology 125 677-687, 2003

44. Schwirzke M, Evtimova V, Burtscher H, Jarsch M, Tarin D and Weidle UH: Identification of metastasis-associated genes by transcriptional profiling of a pair of metastatic versus nonmetastatic human mammary carcinoma cell lines. Anticancer Res 21: 1771-1776, 2001

45. Alpan RS, Sparvero S and Pardee AB: Identification of mRNAs differentially expressed in quiescence or in late G1 phase of the cell cycle in human breast cancer cells by using the differential display method. Mol Med 2: 469-478, 1996.

46. Zambon A, Mandruzzato S, Parenti A, et al: MAGE, BAGE, and GAGE gene expression in patients with esophageal squamous cell carcinoma and adenocarcinoma of the gastric cardia. Cancer 91: 1882-1888, 2001

47. Eichmuller S: Towards defining specific antigens for cutaneous lymphomas. Onkologie 25: 448-454, 2002.

48. Melloni G, Ferreri AJ, Russo V, et al: Prognostic significance of cancer-testis gene expression in resected non-small cell lung cancer patients. Oncol Rep 12: 145-151, 2004.

49. De Backer O, Arden KC, Boretti M, et al: Characterization of the GAGE genes that are expressed in various human cancers and in normal testis. Cancer Res 59: 3157-3165, 1999.

50. Berglund P, Stighall M, Jirstrom K, et al: Cyclin E overexpression obstructs infiltrative behavior in breast cancer: a novel role reflected in the growth pattern of medullary breast cancers. Cancer Res 65: 9727-9734, 2005 